\title{
Performance comparison between 802.11 and 802.11p for high speed vehicle in VANET
}

\author{
Mohammed Hasan Alwan', Khairun N. Ramli², Yasir Amer Al-Jawher ${ }^{3}$, Aws Zuhair Sameen ${ }^{4}$, \\ Hussain Falih Mahdi ${ }^{5}$ \\ ${ }^{1,2,3}$ Department of Communication, Faculty of Electrical and Electronic, University Tun Hussein Onn Malaysia, Malaysia \\ ${ }^{4,5}$ Department of Electrical, Electronics and Systems, Faculty of Engineering and Built Environment, \\ Universiti Kebangsaan Malaysia (UKM), Malaysia
}

\begin{tabular}{l}
\hline \hline Article Info \\
\hline Article history: \\
Received Aug 28, 2018 \\
Revised Apr 11, 2019 \\
Accepted Apr 25, 2019 \\
\hline
\end{tabular}

\section{Keywords:}

$802.11 \mathrm{p}$

Mac protocol

Mobility

Performance evaluation

Speed

Sumo

Vehicular ad hoc networks

\begin{abstract}
Vehicular ad-hoc networks (VANETs) technology has been emerged as a critical research area. Being ad-hoc in nature, VANET is a type of networks that is created from the concept of establishing a network of cars for a specific need or situation. Communication via routing packets over the highspeed vehicles is a challenging task. Vehicles mobility, speed can vary depending on the road specification. However, on highway, the speed can be increase up to $120-200 \mathrm{Km} / \mathrm{H}$. Moving at high speed can affect the efficiency of data delivery. In particular V2I traffic where moving car trying to deliver data to fixed space units which are designed to collect and process data from vehicles. Different protocols have been proposed to be implemented for VANET infrastructure, including 802.11 and 802.11p. The performance of these proposed protocols has not been compared and investigated to find out the robust mechanism for handling high speed VANETs. In this paper, the performance of the most widely deployed MAC protocols for handling wireless communication which is 802.11 and the 802.11p have been compared, which is a customized version for high speed modes. Performance is investigated in term of data delivery evaluation metrics including network throughput, delay and packet delivery ration. Results show that $802.11 \mathrm{p}$ has efficiently enhanced the network performance where network throughput is increased, delay is decreased, and packet delivery ratio is increased as well.
\end{abstract}

Copyright $@ 2019$ Institute of Advanced Engineering and Science. All rights reserved.

\section{Corresponding Author:}

Mohammed Hasan Alwan,

Department of Communication, Faculty of Electrical and Electronic

University Tun Hussein Onn Malaysia,

Batu Pahat, Johor, Malaysia.

Email: mohammed.h.alrubaye@ieee.org

\section{INTRODUCTION}

Recently, Vehicular Ad-hoc Networks (VANET) becomes more popular and widely deployed over all the roads across the world. Most of modern cars are equipped with Wireless modules which provides vehicles to communicate with each other's and with communication control points [1]. Enhancing Inter-Vehicle communication and roadside communication are considered as the most popular wireless communication research topic. VANET allows road vehicles to notify other vehicles about traffic jams, sudden stops and other hazardous road conditions [2]. The huge number of expected benefits of VANET and a number of supporting vehicles are likely become the most realized implementation of mobile Ad hoc networks. Short range IEEE 802.11 can be used for vehicle communications using suitable radio interface technology [3]. However, a new standard for both physical and MAC layer has been developed to meet the requirement of communication between vehicles, IEEE $802.11 \mathrm{p}$ is an approved amendment to the IEEE 
802.11 standard which provides Wireless Access in Vehicular Environments (WAVE) [4]. The enhancements that are applied to 802.11 support the applications of the Intelligent Transportation Systems [5].

The navigation systems availability of each vehicle allows it to recognize the geographic location in addition to its neighbors. On the other hand, Geographic Routing which is a specific type of routing approach, becomes possible where choosing a neighbor who is geographically closer to that destination where frames are simply forwarded to a destination. With the vehicles, rapid growth and increasing number of roadside traffic monitors, the enhancement of navigation systems, the wireless network devices low cost promising peer-to-peer (P2P) applications and externally driven services to vehicles became available. For this purpose, the Wireless Access in Vehicular Environments (WAVE) standards is proposed by the Intelligent Transportation Systems (ITS) to define an architecture which enables vehicle-to- vehicle (V2V) and vehicle-to-infrastructure (V2I) wireless communications collectively [6]. Figure 1 shows the general VANET network communication.

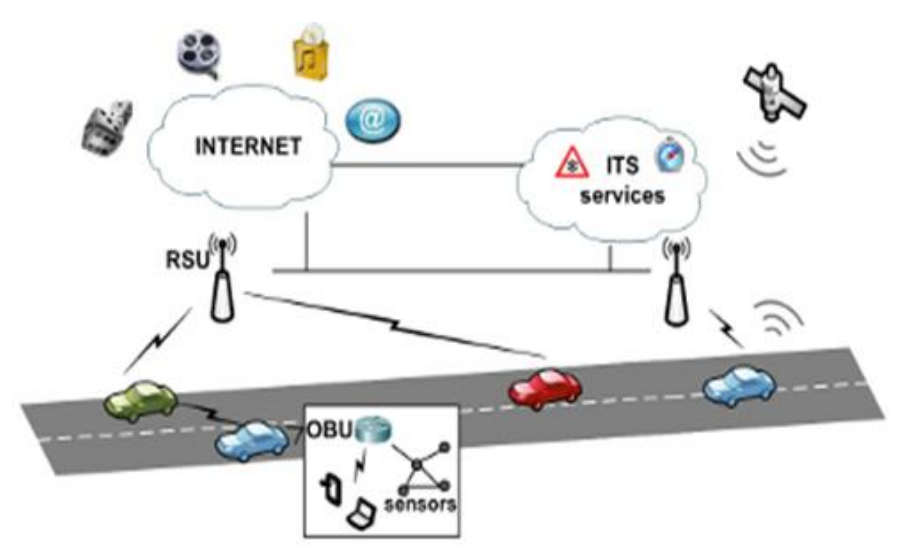

Figure 1. VANET network communication [6]

In this research, the performance of 802.11 and $802.11 \mathrm{p}$ performance in a high-speed vehicle environment have been compared and to investigate how they performed to find out how 802.11 and $802.11 \mathrm{p}$ are performing when vehicle speed changing and how can this reflect in term of communication performance. The mechanism of data delivery can be affected in case of frequent distance changes away from the road side unit where data is delivered. Different vehicles speed needs to be measured to cover a wide range of vehicles possibilities.

The rest of this paper is organized as follows: In section 2 related work is presented. Section 3 illustrated the simulation scenarios for data delivery performance evaluation. Section 4 illustrate the measurement results and analysis; Finally, a conclusion is presented in section 5.

\section{RELATED WORK}

In VANET, different issues are considered as critical and particularly for safety application where data delivery is critical and may threat human lives. $802.11 \mathrm{p}$ protocol has been proposed to provide more reliable data communication in high speed nodes environment. Demmel et al. note that latencies have not been investigated in these previous studies, when they are supposed to be a major improvement brought upon by the amendment [7]. Furthermore, the whole set of possible speed classes has not been investigated where each study focuses on a precise range such as lower speeds or freeway speeds [8]. Experimental conditions are not very well known or controlled, notably for being open. Although it is advantageous to measure performance in real road conditions, as they have shown to be very variable depending on the experimental environment, it is important to obtain baseline performance details in more controlled environments first. propose a detailed post processing analysis of performance metrics (latency, range and frame loss), for measurements taken in a controlled, representative freeway-like environment and using the full range of speeds achieved by motor vehicles [9].

Gräfling et al. provide performance evaluation for using dedicated short-range communication standard (DSRC) in data delivery for VANET communication [10]. The new DSRV standard solution provide a multi-channel which can server multiple application types and provide high performance for future 
VANET communication. Performance evaluation includes different communication parameters including throughput, collision probability and delay. Different scenarios have been built to simulate different scenarios and analyze results [11]. Performance evaluation shows that new standards can prioritize data packets for dense and loaded scenarios where network throughput has been decreased significantly and delay has been increased.

Mobility impact in IEEE 802.11p infrastructure less vehicular networks. Due to high mobility, two dynamic contention window mechanisms to alleviate network performance degradation. The first scheme provides a dynamic level of service priority via adaptation to the number of neighboring nodes, while the second scheme provides service priority based on node relative speed. Extensive simulation results demonstrate a significant impact of mobility on the IEEE 802.11p MAC performance where the unfairness problem with the vehicle-to-vehicle $(\mathrm{V} 2 \mathrm{~V})$ communications and the effectiveness of the proposed MAC schemes [12].

Performance evaluation of IEEE 802.11p enabled vehicular video surveillance system. [13] assess the feasibility of such system by analyzing the video end-to-end distortion for a target vehicle, located several hops away from the gateway, when it is alone or there are also other vehicles transmitting video [14]. They demonstrate the importance of dynamic adaptation of the video bit rate of each vehicle depending on the number and positions of the participating vehicles.

A performance evaluation for VANET using LTE and IEEE 802.11p has been proposed in [15]. A detailed study of performance evaluation for both standards for different parameters configuration including vehicle density and transmission frequency have been done in [16]. Different performance evaluation considerations have been checked including reliability, mobility support, delay and scalability for different VANET application context [17]. Intensive simulation experiments have been implemented to evaluate the standard's effectiveness. Results indicate that $802.11 \mathrm{p}$ provides acceptable performance for sparse network topologies. On the other hand, LTE meets most of the application requirements in terms of reliability, scalability and mobility support. However, it is challenging to obtain stringent delay requirements in the presence of higher cellular network traffic load.

\section{SIMULATION TOPOLOGY}

To evaluate the performance of VANET, a realistic simulation environment is needed to be built. In this study Wilmington, New Castle County, Delaware, 19801, United States of America. The coordination of the used map is $-75.5875 *-75.5348 \mathrm{H}$ and 39.7272 / 39.7096 V. has been selected. Figure 2 shows the real map of selected highway.

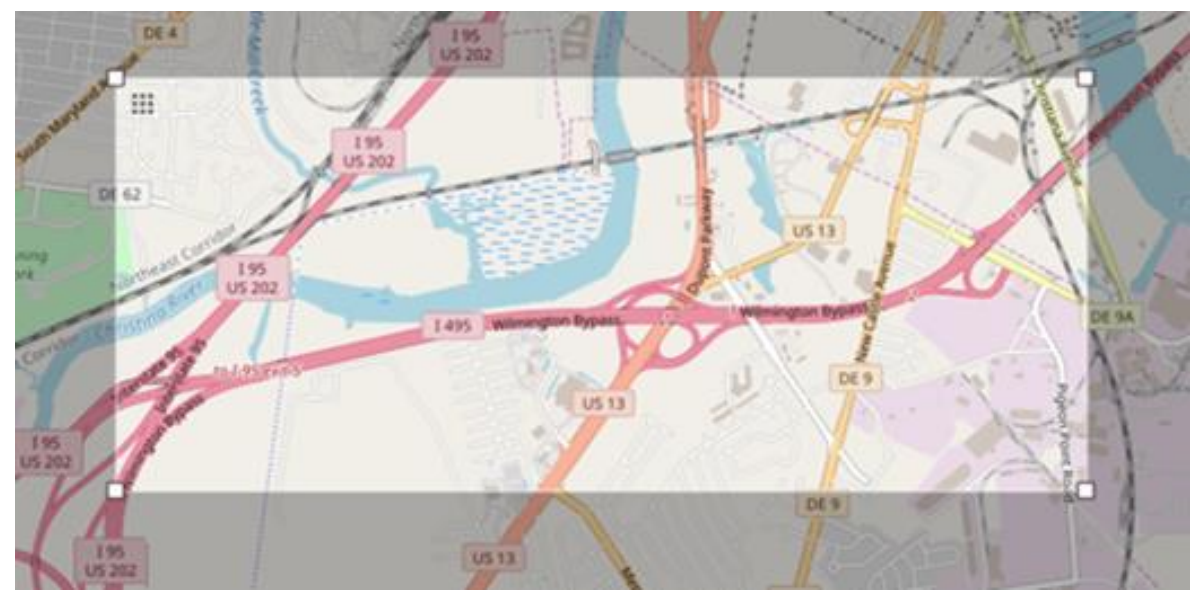

Figure 2. High speed way, Wilmington simulation map

The open street map (OSM) project has been utilized [18]. OSM is a collaborative project to create a free editable map of the world. The creation and growth of OSM have been motivated by restrictions on the use or availability of map information across much of the world. The advent of inexpensive portable satellite navigation devices. Rather than the map itself, the data generated by the OSM are considered as a primary output. The data is then available for use in traditional applications. 
Then the exported OSM files are input to the SUMO simulator to generate traffic simulation [19]. The first step in SUMO is to generate the traffic network where vehicles are moving in high speed. After that the vehicle trips are generated by specifying the required time of the simulation. Finally, SUMO creates a complete simulation file which includes all vehicles, paths and trip details for each vehicle depending on the provided map.

A python-based script is then used to translate the SUMO simulation file to the NS2 mobility and configuration files. In the NS2 simulator the mobility and the speed of vehicles are adjusted in the mobility file. Data are forwarded to the central communication location for storing and processing. Figure 3 illustrate the location of the vehicles in the simulating network before inserting the road side unit (RSU)s. A grid of road side units is inserted in the network to cover the whole simulation area as shown in Figure 4.

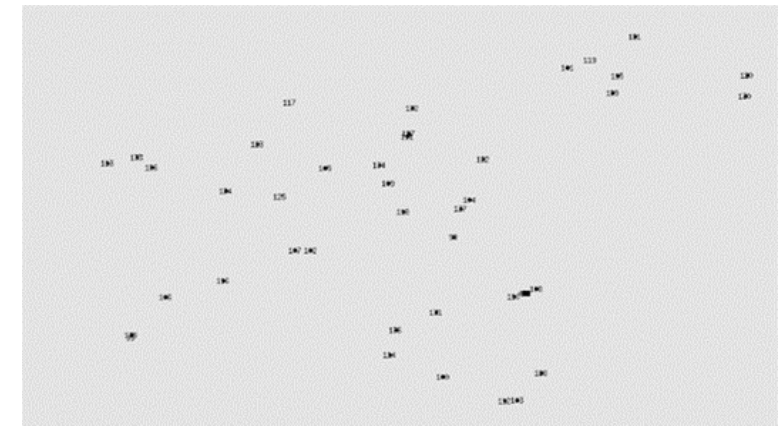

Figure 3. The NS2 simulation network before adding RSUs

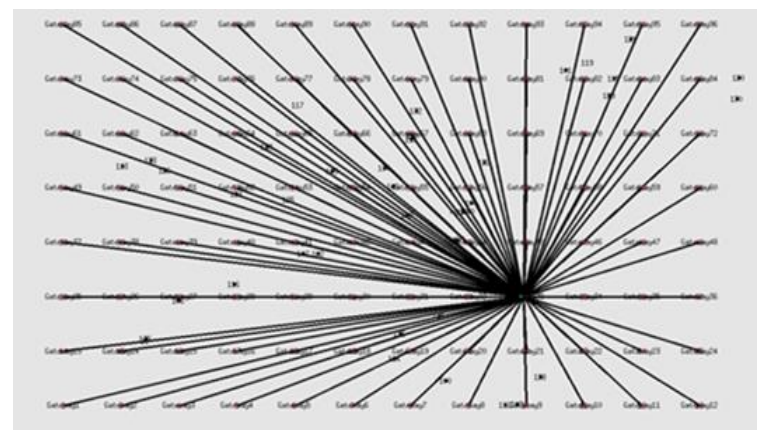

Figure 4. The simulation network after adding RSUs

\subsection{Simulation parameters}

The network simulation parameters that are assigned to network nodes are listed in Table 1 where different simulation scenarios have been implemented using different simulation time, different vehicle speeds have been simulated and performance comparison has been investigated [20].

Table 1. Netwrok simulation parameters values

\begin{tabular}{cc}
\hline Parameter & Value \\
\hline Channel type & Wireless Channel \\
Radio-propagation model & Two Ray Ground \\
Network Interface Type & Wireless Phy. \\
Antenna type & Omni Antenna \\
Interface queue type & Drop Tail / Pri-Queue \\
Maximum packet in Queue & 50 \\
MAC type & $802.11,802.11 \mathrm{p}$ \\
Topographical Area & $13000 \times 13000 \mathrm{sqm}$ \\
Routing protocols & DSDV \\
Number of mobile nodes & 40 \\
Simulation Time & $60 \mathrm{~seconds}$ \\
Data Flow & CBR \\
Packet Size & $256 \mathrm{byte}$ \\
Data Bit rate & $0.5 \mathrm{MB} / \mathrm{s}$ \\
Vehicle speed &
\end{tabular}

\subsection{Performance metrics}

Different evaluation metrics can be measured, including:

\section{End to end delay}

The end-to-end delay represents the average amount of time, which is taken by each vehicle packets to traverse the path from a vehicle to another vehicle or RSU. E2E delay is accumulative of different network delay types. It mainly includes processing delay for buffering delay, route discovery and intermediate node forwarding delay. E2E delay is considered as a significant network performance indicator where small values E2E delay represents better network performance. Increased delay can be caused by routing overhead or link failure. To estimate the average E2E delay for a network, the following equation is used: 


$$
T_{E 2 E}=\frac{\left(T_{R}-T_{S}\right)}{\text { number of packets }}
$$

Where, T_E2E is the Average E2E Delay, TR is packets received time at destination node and TS is packets sent time from the source node.

\section{Packet loss}

Packet loss is obtained by subtracting the number of packets received at Access Point from the total number of packets transmitted.

$$
\begin{aligned}
& \text { Packet lost }=\sum \text { transmitted Packets }-\sum \text { received Packets } \\
& \text { Packet loss Ratio }=\frac{(\text { Packets losts } * 100)}{\sum \text { transmitted Packets }}
\end{aligned}
$$

\section{Network throughput}

Throughput referred to the vehicles packets number which is correctly delivered over the network for a specific time. To measure throughput put in a link between two nodes total number of packets that have been successfully delivered to the desired nodes are counted. Increasing throughput values is a great indicator for enhanced performance. It is measured in bits per second (bit/s or bps). Throughput can be represented mathematically as in equation below:

$$
\text { Throughput }=\frac{\text { no. of delivered packet } * \text { packet size } * 8}{\text { total simulation time }}
$$

\section{MEASUREMENT RESULTS AND ANALYSIS}

To investigate the performance of both 802.11 and $802.11 \mathrm{p}$, we have illustrated the performance comparison between both protocols in term of evaluation metrics. The estimated results of the experimented various main scenarios of different speeds are shown in the following diagrams. Figure 5 shows that $802.11 \mathrm{p}$ has decline the number of dropped packets significantly when it is compared with 802.11 when the vehicle speed is increased, which means that the $802.11 \mathrm{p}$ has been enhanced the number of dropped packets up to $70 \%$. The efficient enhancement in number of dropped packets for $802.11 \mathrm{p}$ is acheived by handling performance anomaly problem efficiently.

The network delay results show better performance for $802.11 \mathrm{p}$ where the end to end delay has been decreased up to $12 \%$ less than the results of 802.11 mac protocol as shown in Figure 6. 802.11p allow faster delivery of packets for its destination in higher speed vehicles due to better handling of traffic flow and signals power for mobile devices.

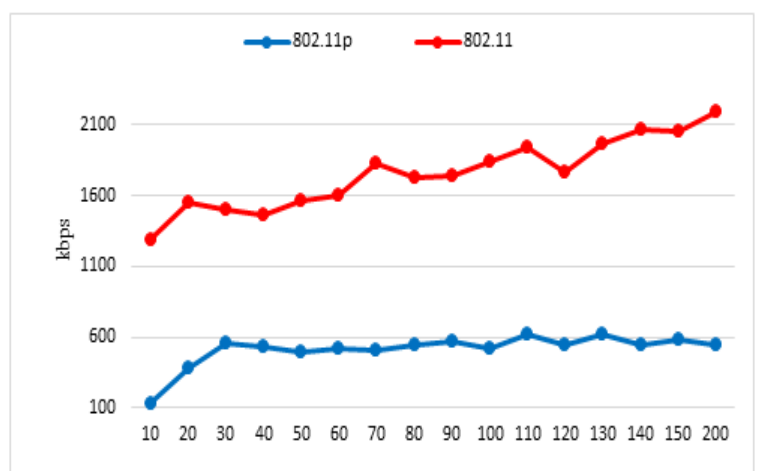

Figure 5. Number of dropped packets for both 802.11 and $802.11 \mathrm{p}$

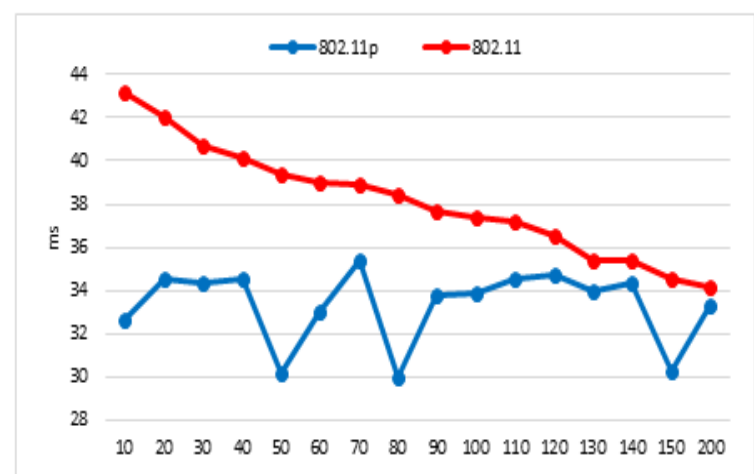

Figure 6. End to end delay for both 802.11 and $802.11 p$ 
802.11p also enhance the network performance where the number of successfully received packets is increased as shown in Figure 7. The network throughput has been increased up to $41 \%$ more than 802.11 mac protocol. Effeceint handling of performance anomaly problem by $802.11 \mathrm{p}$ has dramarically decrease the number of dropped packet and thus enhance the delivery of data traffic to its final destination successful handling of data delivery reflected in better network throughput and higher packet delivery ratio as dipcted in both Figures 7, 8. Figure 7 shows that the packet delivery ratio of the $802.11 \mathrm{p}$ mac protocol is much larger than the packet delivery ratio of 802.11 . The packet delivery ratio has been enhanced up to $41 \%$ of the 802.11 .

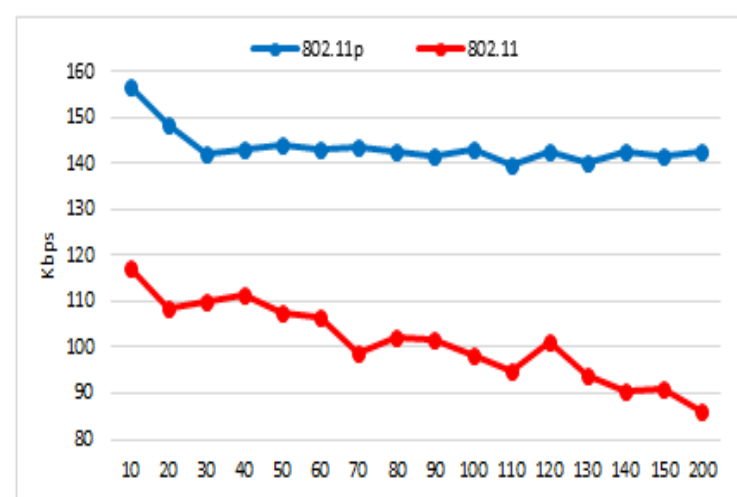

Figure 7. Network throughput for both 802.11 and $802.11 \mathrm{p}$

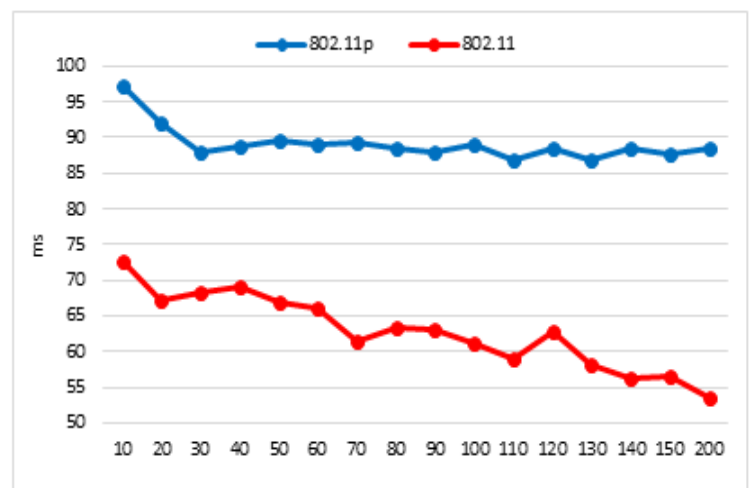

Figure 8. PDR for both 802.11 and $802.11 \mathrm{p}$

The results show performance degradation for data communication when vehicle speed is increased which indicates that data communication is affected in high vehicles speed mobility. The performance has been measured in term of evaluation metrics, including throughput, end to end delay, dropped packets and packet delivery ratio. $802.11 \mathrm{p}$ mac protocols provide better performance than 802.11 where it can handle data communication more efficiently where the performance metrics is enhanced. The number of dropped packets is decreased up to $70 \%$, where the packet delivery ratio and network throughput is increased up to $41 \%$. The end to end delay is also decreased down to $12 \%$.

Performance anomaly and particularly in higher speed vehicles, mobility is still a critical performance issue, despite the enhancement of $802.11 \mathrm{p}$ protocol where the number of dropped packets is increased when the vehicle speed is increased resulting in performance degradation and data loss. The objective of investigating the effects of high speed vehicles and its consequences on performance anomaly problem since $802.11 \mathrm{p}$ is accomplished were results show a direct performance degradation of data communication due to increased vehicle speeds in term of evaluation metrics for both 802.11 and $802.11 \mathrm{p}$ mac protocols, however 802.11 p provides better performance than 802.11 protocols.

\section{CONCLUSION}

This paper present performance comparison for using 802.11 and 802.11p in VANET for high speed vehicle mobility model. A real environment based simulation scenario has been built where different vehicle speed have been simulated, to evaluate the performance various data delivery, evaluation metrics have been measured including network throughput, network relay and packet delivery ratio. Results show that $802.11 \mathrm{p}$ protocols provide better performance in term of evaluation metrics where $802.11 \mathrm{p}$ has reduced the number of dropped packets significantly, results show that $802.11 \mathrm{p}$ has enhanced the number of dropped packets up to $70 \%$. Also, the end to end delay has been decreased up to $12 \%$ less than the results of 802.11 . The packet delivery ratio has been enhanced up to $41 \%$ of the 802.11 as well.

\section{REFERENCES}

[1] M. S. Anwer and C. Guy, "A survey of VANET technologies," J. Emerg. Trends Comput. Inf. Sci, vol. 5, p. 671, 2014.

[2] M. Altayeb and I. Mahgoub, "A survey of vehicular ad hoc networks routing protocols," International Journal of Innovation and Applied Studies, vol. 3, pp. 829-846, 2013. 
[3] C. Cooper, et al., "A comparative survey of VANET clustering techniques," IEEE Communications Surveys \& Tutorials, vol. 19, pp. 657-681, 2017.

[4] IEEE, "IEEE 802.11p - IETF," ed, 2010.

[5] I. Al-Anbagi and H. T. Mouftah, "WAVE 4 V2G: Wireless access in vehicular environments for vehicle-to-grid applications," Vehicular Communications, vol. 3, pp. 31-42, 2016.

[6] S. Al-Sultan, et al., "A comprehensive survey on vehicular ad hoc network," Journal of network and computer applications, vol. 37, pp. 380-392, 2014.

[7] S. Demmel, et al., "Empirical IEEE 802.11 p performance evaluation on test tracks", Intelligent vehicles symposium (IV), IEEE, pp. 837-842, 2012.

[8] W. Alasmary and W. Zhuang, "Mobility impact in IEEE 802.11 p infrastructureless vehicular networks," Ad Hoc Networks, vol. 10, pp. 222-230, 2012.

[9] B. Bellalta, et al., "Performance evaluation of IEEE 802.11 p-enabled vehicular video surveillance system," IEEE Communications Letters, vol. 18, pp. 708-711, 2014.

[10] S. Gräfling, et al., "Performance evaluation of IEEE 1609 WAVE and IEEE $802.11 \mathrm{p}$ for vehicular communications," Second Internation Conference on Ubiquitous and Future Networks (ICUFN), pp. 344-348, 2010.

[11] Kiho Lim and D. Manivannan, "An efficient protocol for authenticated and secure message delivery in vehicular ad hoc networks", Vehicular Communications, Elsevier, pp 30-37,2016.

[12] N. Singh, R. L. Dua, and V. Mathur, "Network simulator NS2 2.35", International Journal of Advanced Research in Computer Science and Software Engineering, vol. 2277, pp. 224-228, 2012.

[13] Adel Mounir Said, et al., "Modeling interactive real-time applications in VANETs with performance evaluation\|", Computer Networks, pp 66-78, 2016.

[14] N. S. Ravindranath, et al., "Performance Evaluation of IEEE 802.11ac and 802.11n using NS3", Indian Journal of Science and Technology, pp 1-9, 2016.

[15] Z. H. Mir and F. Filali, "LTE and IEEE 802.11 p for vehicular networking: a performance evaluation," EURASIP Journal on Wireless Communications and Networking, vol. 2014, p. 89, 2014

[16] Steffen Moser, et al., "MIMO-Enabling PHY Layer Enhancement for Vehicular Ad-Hoc Networks", Wireless Communications and Networking Conference, IEEE, pp142-147, 2015.

[17] Sai Al-Sultan, et al., "A comprehensive survey on vehicular Ad Hoc network\|", Journal of Network and Computer Applications, pp380-392, 2014.

[18] openstreetmap. Available: https://www.Openstreetmap.org, 2018.

[19] M. Behrisch, L. Bieker, J. Erdmann, and D. Krajzewicz, "SUMO-simulation of urban mobility: an overview," $3^{\text {rd }}$ International Conference on Advances in System Simulation Proceedings of SIMUL 2011, 2011.

[20] Muhammad Sajjad Akbar, et al., "Evaluation OF IEEE 802.11n for Multimedia Application in VANET", 4th International Symposium on Frontiers in Ambient and Mobile Systems, Elsevier, pp 953-958, 2014.

\section{BIOGRAPHIES OF AUTHORS}

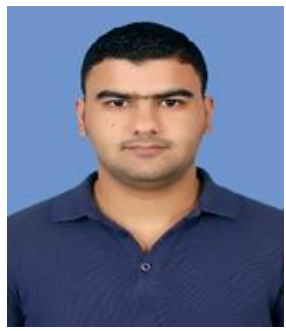

Mohammed Hassan Alwan received his Bachelor in Power and Machine Engineering from the Department of Power and Machine Engineering,Faculty ofEngineering,University of Diyala in 2012. He decides to pursue his master's in communication and Computer Engineering from the Department of Electrical, Electronic and Systems, Faculty of Engineering, Universiti Kebangsaan Malaysia (UKM). He graduated in July 2016. He is now doing PhD.in Electrical Engineering at the Department of Electrical, Electronics and Systems, Faculty of Engineering, (UTHM). His main research area is Communication Engineering on the VANET. He always learned to be under pressure at work.

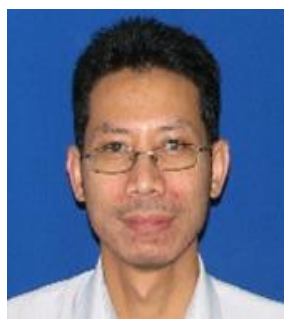

K. N. Ramli was born in Batu Pahat, Johor, Malaysia, in 1974. He obtained a BEng degree in Electronic Engineering from the University of Manchester Institute of Science and Technology (UMIST), United Kingdom in 1997, and then MEng in Universiti Kebangsaan Malaysia (UKM), Malaysia in 2004. Then from 2007 he worked within the Antennas and Applied Electromagnetic research group at University of Bradford, United Kingdom on a number of projects, concentrating on antenna design and computational electromagnetics. He received a $\mathrm{PhD}$ degree for research in electromagnetic problems using hybrid computational methods from the University of Bradford, United Kingdom in 2011. HE is currently working at the Faculty of Electrical and Electronic Engineering, U Universiti Tun Hussein Onn Malaysia (UTHM). His main interests are in the field of computational electromagnetics, antennas, and wireless communications. 

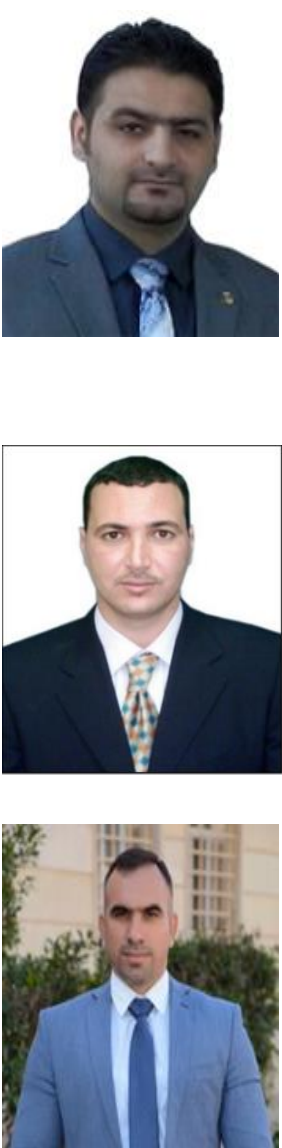

Aws Zuhair Sameen received his bachelor's in Computer and Software Engineering from Department of Computer and Software Engineering, Faculty of Engineering, University of Diyala in 2011. He decides to continue his master's in communication and Computer Engineering in Department of Electrical, Electronic and Systems Engineering, Faculty of Engineering and Built Environment, Universiti Kebangsaan Malaysia (UKM). He graduated in March 2015. He is now doing PhD. in Electrical Engineering at the Department of Electrical, Electronics and Systems Engineering, Faculty of Engineering and Built Environment, Universiti Kebangsaan Malaysia (UKM). His main research area is Biomedical Engineering on BioSignal processing for healthcare technology. He routinely using System Identification techniques for characterization of the human vascular system investigated with optoelectronic BioSignals.

Yasir Amer Jawhar received his BS degree in electrical engineering from the College of Engineering, University of AL-Mustansiriya, Baghdad, Iraq, in 1998, and his MS degree in Engineering from the Faculty of Electrical and Electronic Engineering, University Tun Hussein Onn Malaysia, Johor, Malaysia, in 2015. He is currently working toward his $\mathrm{PhD}$ degree in Communication Engineering from the University Tun Hussein Onn Malaysia. His main research interests are signal processing in communication, OFDM, PAPR reduction in Multicarrier system, 5G waveform design, and wireless network.

Hussain Mahdi is from Malaysia Section, a Ph.D. in electrical engineering at the Electronic Department, Faculty of Engineering, Universiti Kebangsaan Malaysia (UKM). He joined IEEE 7 years ago, and since then. He was the social activity Chair 2012, vice chair 2013 and the chair of IEEE UKM Student Branch 2014. He was IEEE Malaysia Section Student Representative 2015, IEEE Region 10 Asia Pacific Students Activities Committee 2015, IEEEXtreme 9.0 Ambassador 2015, IEEE Day Ambassador 2015, IEEE madC ambassador coordinator of Region 10 2016, IEEEXtreme PA Lead, Region 10 and ambassador coordinator 2016 , IEEE Region 10 Student activity committee South-East Asia coordinator 2016, IEEE Malaysia Section Young Professional Humanitarian activities Chair 2016,2017, IEEE Region 10 Young Professional Committee South-East Asia coordinator (2017-2018), IEEE Region 10 Humanitarian activities committee 2017. 